\title{
WWOX Suppresses Cell Growth and Induces Cell Apoptosis via Inhibition of P38 Nuclear Translocation in Cholangiocarcinoma
}

\author{
Mei Wang ${ }^{a}$ Yongmei Lia Meihong Wu Wei Wang ${ }^{a}$ Biao Gong ${ }^{b}$ Yajie Wang \\ aDepartment of Oncology, Changhai Hospital, Second Military Medical University, Shanghai, China, \\ 'Department of Gastroenterology, Ruijin Hospital, Shanghai Jiao Tong University School of Medicine, \\ Shanghai, PR China
}

\section{Key Words}

Cholangiocarcinoma $[C C A] \cdot$ Extrahepatic cholangiocarcinoma $[$ EHCC] $\bullet$ WWOX • P38 MAPK

\begin{abstract}
Background: Extrahepatic cholangiocarcinoma [EHCC] is the second most common primary hepatic malignancy, and is associated with high morbidity and mortality. We previously reported the decreased expression of WWOX in EHCC samples, but the underlying mechanism remained unclear. Methods: Immunoprecipitation and immunofluorescence were performed to examine the interaction of WWOX and P38 MAPK. Western blot was carried out to detect the expression of ATF2 and eIF-4E. MTT, colony formation, and Annexin V-FITC assays were performed to detect the cell proliferation and apoptosis. IHC was performed to detect the protein expression in clinical samples. Results: WWOX interacted with P38 and modulated its sub-cellular localization, leading to the cytoplasmic retention of P38. WWOX over-expression inhibited the phosphorylation of ATF2 and eIF-4E, while exogenous P38 reversed this reduction in phosphorylation. Ectopic expression of WWOX in EHCC cells led to inhibited proliferation and stimulated apoptosis in a P38 MAPK-dependent manner. In addition, we found a negative association of WWOX with nuclear localization of P38 and expression of phosphorylated ATF2 in EHCC samples. Conclusion: Our data demonstrated the role of WWOX in EHCC progression, revealing the potential of WWOX/p-ATF2 as a novel diagnostic and prognostic marker, and therapeutic target for EHCC.
\end{abstract}

Copyright @ 2014 S. Karger AG, Basel

M. Wang and Y. Li contributed equally to this work.

Yajie Wang,

or Biao Gong
168 Chang Hai Road, Shanghai, 200433 (PR China)

or 197 Rui Jin Second Road, Shanghai, 200025 (PR China)

E-Mail dryajiewang@163.com, E-Mail biaogong@163.com 


\section{Introduction}

Cholangiocarcinoma [CCA] is highly malignant carcinoma that originates from cholangiocytes in the biliary epithelium. CCA can be broadly classified as intrahepatic or extrahepatic based on the anatomic location of the tumor $[1,2]$. CCA accounts for only $3 \%$ of gastrointestinal tumors, but the morbidity and mortality of CCA, particularly extrahepatic cholangiocarcinoma [EHCC], are increasing worldwide [3, 4]. In Asia, EHCC is a challenging disease because of difficulties in diagnosis, treatment, and prognosis. Most EHCC patients are diagnosed in the late stages of the disease. Because of this late diagnosis, the effects of treatment are less than satisfactory, resulting in a median survival of less than 24 months [5, 6]. Thus, there is an urgent need to find effective targets for the treatment of EHCC.

WW domain containing oxidoreductase [WWOX], a gene that spans the second most common chromosomal fragile site [FRA16D], has essential roles in maintaining genomic stability and suppressing tumorigenesis $[7,8]$. WWOX encodes a 414 amino acid protein and possesses two typical N-terminal WW domains, a C-terminal short-chain dehydrogenase reductase [SDR] domain, and a nuclear localization sequence [NLS]. The first $\mathrm{N}$-terminal WW domain is responsible for the classical WW-PPXY interaction, by which WWOX regulates the function of its binding partner and participates in signaling transduction [9]. An accumulating body of evidence has demonstrated deficiencies in WWOX expression in various cancers, including breast cancer, prostate cancer, non-small cell lung cancer, renal cell carcinoma, and pancreatic adenocarcinoma [10-15]. Introduction of WWOX into cancer cells leads to the suppression of cell proliferation, a result that occurs because WWOX attenuates the function of onco-proteins by sequestering in the cytoplasm through a direct interaction and preventing their nuclear translocation. Examples of these WWOX interacting proteins include activator protein $2 \gamma$ [AP-2 $\gamma$ ], p73 [p53 homologue], erythroblastic leukemiaviral oncogene homolog 4 [ErbB4], c-Jun, and Runt-related transcription factor 2 [RUNX2] [16-24].

P38 mitogen-activated protein kinase [MAPK] belongs to the mitogen-activated protein [MAP] family of kinases, and is activated by numerous physical and chemical stresses including oxidative stress, osmotic shock, cytokines, and growth factors. The activation of P38 MAPK is mediated by dual phosphorylation of the T-P-Y motif by MAPK kinase 4 [MKK4] and MKK3/6. Upstream of the MKKs are MAPKKKs, including MEKKs 1-4, which are members of the mixed-lineage kinases [MLK3] and ASK-1 [25-27]. Once activated, P38 MAPK translocates from the cytosol to the nucleus, where it phosphorylates serine/ threonine residues on substrates involved in various cellular responses [25]. Over-activation of p38 MAPK is found in prostate cancer, breast cancer, bladder cancer, liver cancer, lung cancer, transformed follicular lymphoma, and leukemia, and it contributes to processes such as the epithelial-mesenchymal transition [EMT] of primary cancer cells, the acquisition of invasive and migratory capabilities, and the extravasation of migrating tumor cells $[28,29]$.

In our previous study, we found that WWOX expression is deficient in EHCC tissues [30], and that WWOX may be involved in EHCC tumorigenesis. However, the underlying mechanism was still poorly-understood. Here, we demonstrated that WWOX could interact with P38 MAPK and modulated its sub-cellular localization, resulting in the inhibition of ATF2 and eIF-4E phosphorylation. Over-expression of WWOX in EHCC cells led to inhibition of proliferation and stimulation of apoptosis. Finally, we found a negative association between WWOX and nuclear P38/ phosphorylated ATF2 in EHCC samples. Thus, our results revealed the mechanism of EHCC progression and provided potential therapeutic targets for the treatment of EHCC.

\section{Materials and Methods}

\section{Cell cultures}

QBC939 cholangiocarcinoma cells were cultured in RPMI-1640 medium [Invitrogen, CA, USA] supplemented with 10\% fetal bovine serum [FBS]. RBE and HuCCA-1, cells were cultured in Dulbecco's modified essential medium [DMEM, Invitrogen, CA, USA] supplemented with 10\% FBS. HEK293 cells 
were cultured in DMEM [Invitrogen, CA, USA] supplemented with 10\% FBS. All cells were maintained in a humidified atmosphere with $5 \% \mathrm{CO}_{2}$ at $37^{\circ} \mathrm{C}$.

\section{Plasmids}

The P38 plasmid was obtained from ADDGENE. The plasmid containing a mutated variant of P38 was generated using a QuikChange $囚$ Multi Site-Directed Mutagenesis Kit [Stratagene, USA]. The mutant plasmid encoded the P38 gene containing a "PPAY" to "LLVL" mutation at the amino acid 242. To generate the WW OX plasmid, WWOX cDNA was amplified by PCR and inserted into the PCMV-HA vector [Promega, Madison, WI, USA] using Kpn I and Xho I. The plasmid containing a mutant WWOX was similarly generated using PCR. This mutant contained a deletion of the first WWOX domain.

\section{Immunohistochemistry}

With the approval of the Ethics Committee of Changhai Hospital, thirty extrahepatic cholangiocarcinoma and five normal bile duct samples [acquired between 2008-2013] were obtained from the paraffin archives of our hospital, For immunohistochemical analysis, endogenous peroxidase activity was blocked using $3 \%$ $\mathrm{H}_{2} \mathrm{O}_{2}$ for 2 hours, followed by incubation with $5 \%$ normal goat serum and then primary antibody overnight at $4^{\circ} \mathrm{C}$. Immunohistochemistry was scored by three investigators according to the following scale: 1 , negative; 2, minimal; 3, moderate; 4, strong; or 5, maximal. The correlation between WWOX and nuclear P38/p-ATF2 was calculated using the analysis of variance test.

\section{Antibodies and Western blot}

For Western blot analysis, cell lysates were separated using 12\% SDS-polyacrylamide gels, transferred to nitrocellulose membranes, and probed with various antibodies. Primary antibodies included: anti-p-P38 [Abcam, ab4822], anti-P38 [ab7952], anti-p-ATF2 [ab28848], anti-ATF2 [ab47476], anti-eIF-4E [ab1126], anti-p-eIF-4E [ab33766], and anti-Bax [ab10813], all obtained from Abcam [MA, USA]. Anti-caspase-3 [sc7272], anti-Bcl-2 [sc-492], and anti-tubulin [sc-53646] were obtained from Santa Cruz [CA, USA]. Anti-flag [CW0287] and anti-HA [CW2027] were obtained from Kangwei Biotechnology [Beijing, China].

\section{Immunoprecipitation}

For coimmunoprecipitation of WWOX and P38, HEK293 cells were transfected with wild or mutant type of Flag-WWOX and HA-P38 plasmids. After $24 \mathrm{~h}$, cells were harvested in ice-cold lysis buffer [10 mM Tris- $\mathrm{HCl}, \mathrm{pH}$ 7.5, $100 \mathrm{mM} \mathrm{NaCl}, 5 \mathrm{mM}$ EDTA, 0.5\% Nonidet P-40, $5 \mathrm{mM} \mathrm{Na} 3 \mathrm{VO} 4,1 \mathrm{mM} \mathrm{NaF}$, and $1 \mathrm{mM}$ phenylmethylsulfonyl fluoride]. After centrifugation, the supernatants were incubated with anti-Flag or anti-HA antibodies at $4^{\circ} \mathrm{C}$ overnight. After extensive washing with lysis buffer, immunoprecipitates were analyzed by immunoblot as described above using anti-HA or anti-Flag antibodies.

\section{Immunofluorescence}

Cells were plated on special $60-\mathrm{mm}$ dishes for con-focal microscopy and treated as described. The cells were then washed with phosphate-buffered saline [PBS] and fixed with $4 \%$ paraformaldehyde and $0.25 \%$ Triton X-100 for $1 \mathrm{~h}$ at room temperature. After blocking for $30 \mathrm{~min}$ at room temperature, the cells were incubated with anti-P38 or anti-WWOX antibodies for $1 \mathrm{~h}$ at room temperature. The samples were then incubated with corresponding fluorescein isothiocyanate [FITC]-conjugated or Cy3-conjugated secondary antibodies for $1 \mathrm{~h}$ at room temperature. The nuclei were stained with 4',6-diamidino-2-phenylindole [DAPI] for $5 \mathrm{~min}$ at room temperature. Images were captured using a Leica laser scanning confocal microscope.

\section{MTT Assays}

Cells were mock-treated or infected with Ad-EGFP, Ad-WWOX, or Ad-WWOX plus Ad-P38. After 24 $\mathrm{h}$, the cells were plated in 96-well plates at a density of 5000 cells/well and incubated in complete media overnight. Cell proliferation was calculated on the following day using the MTT cell viability assay [Promega Corp., Madison, WI] according to the manufacturer's protocol.

\section{Colony Formation Assay}

Cells were mock-treated or infected with Ad-EGFP, Ad-WWOX, or Ad-WWOX plus Ad-P38. After $24 \mathrm{~h}$, the cells were harvested with $0.125 \%$ trypsin/0.05\% EDTA, pelleted, resuspended in $1 \mathrm{ml}$ of fresh media 
with a 22-gauge needle to disperse the cell clumps, and then counted on a hemocytometer using trypan blue. The cells were then plated at limiting dilutions in six-well plates and allowed to adhere overnight. The cultures were incubated for 14 days, harvested, and stained with $0.5 \%$ crystal violet in methanol. Colony number was determined using a dissecting microscope, with a population of more than 20 cells being counted as one colony.

\section{Annexin V-FITC apoptosis assay}

To quantify apoptotic and necrotic cell death induced by over-expression of WWOX or WWOX plus P38, we used the Annexin V-FITC apoptosis detection kit [BD Biosciences], according to the manufacturer's instructions. Briefly, cells in addition with the culture medium were harvested by centrifugation at 1000 rpm for 5 min. After washing with PBS, the cells were stained with annexin V-FITC staining solution for 30 min at room temperature, followed by staining with PI for $10 \mathrm{~min}$ at room temperature.

Statistics

Statistical analyses were performed using two-sample, two-tailed, equal variance Student's t-test. Statistical significance was set at $* \mathrm{P}<0.05$ and ${ }^{* *} \mathrm{P}<0.01$.

\section{Results}

WWOX interactes via its WW1 domain with the P-P-X-Y motif of P38 MAPK

In our previous study, we found that WWOX expression is deficient in EHCC tissues compared to healthy controls. To investigate the relevance of WWOX to EHCC tumorigenesis and explore the underlying mechanism, we searched for possible WWOX-interacting proteins using PepCyber, Uniprot, DOMINO, ELM, PDZbase and the 3DID database. WWOX functions primarily through binding to the P-P-X-Y motif of target proteins via its WW domain, and it acts as a tumor suppressor by modulating the function of target proteins. We indentified P38, an important MAPK involved in EHCC, as one of several P-P-X-Y motif-containing proteins. To investigate the putative interaction between WWOX and P38, we employed wild and mutant types of P38 and WWOX plasmids in our experiments [details of mutation were contained in Materials and Methods]. Co-IP assays were performed using these constructs. As shown in Fig. $1 \mathrm{~A}$ and B, wt-WWOX interacted with wt-P38, while the binding affinity between wt-WWOX and mt-P38 was much lower. Binding of mt-WWOX and wt-P38 was similar with that of wt-WWOX and mt-P38. Furthermore, binding was completely abolished when both WWOX and P38 were mutated. To verify co-localization of WWOX with P38, we used Confocal Laser Scanning Microscopy [CLSM]. As shown in Fig. 1C, both WWOX [red] and p38 [green] localized in the cytoplasm. When cells were under stress, such as during stimulation with IL-6, P38 and WWOX still co-localized in the cytoplasm. Then we employed a normal bile duct cell lines KNBD to detect the endogenous WWOX-P38 binding. As expected, WWOX and P38 co-localized in the cytoplasm. When KNBD cells were treated with IL-6, no obvious nuclear translocalization of P38 was observed. Together, these experiments confirmed that WWOX co-localized with and therefore interacted with P38 in vivo.

WWOX did not alter the expression and phosphorylation state of P38 MAPK, but it did sequester P38 in cytoplasm

After confirming the interaction between WWOX and P38, We next analyzed the effect of WWOX on P38. As shown in Fig. 2A and B, when WWOX was introduced into QBC939 cells, neither expression nor the phosphorylation state of P38 were noticeably changed. Similarly, when the WW1 domain of WWOX was mutated, neither the expression nor the phosphorylation state of P38 was affected. We next investigated the effect of WWOX on P38 under stress conditions. As shown in Fig. 2C and D, phosphorylation of P38 was significantly elevated in RBE cells following treatment with IL-6. However, phosphorylation and expression of P38 were not changed after WWOX was introduced in the presence of IL6. A previous report indicated that WWOX modulates the function of transcription factors 
Fig. 1. WWOX interacted with the P-P-X-Y domain of P38 via its WW domain. HEK293 cells were co-transfected with plasmids containing wild or mutant type WWOX together with wild or mutant type of P38 plasmids. After antibody or anti-HA antibody, [B] followed by immunoblotting with anti-HA [A] antibody or anti-Flag [B] antibody was conducted. Unimmunized mouse IgG was used as a control. [C] HEK293 cells were transfected with P38 plasmid or P38 plasmid plus WWOX plasmid, and $24 \mathrm{~h}$ later, the cells were treated with IL-6 or left untreated for an additional $6 \mathrm{~h}$, followed by processing for immunofluorescence. [D] KNBD cells were treated with IL-6 or left untreated for $6 \mathrm{~h}$, followed by processing for immunofluorescence. Bar $=10 \mu \mathrm{m}$. 24h, immunoprecipitation with anti-Flag [A]

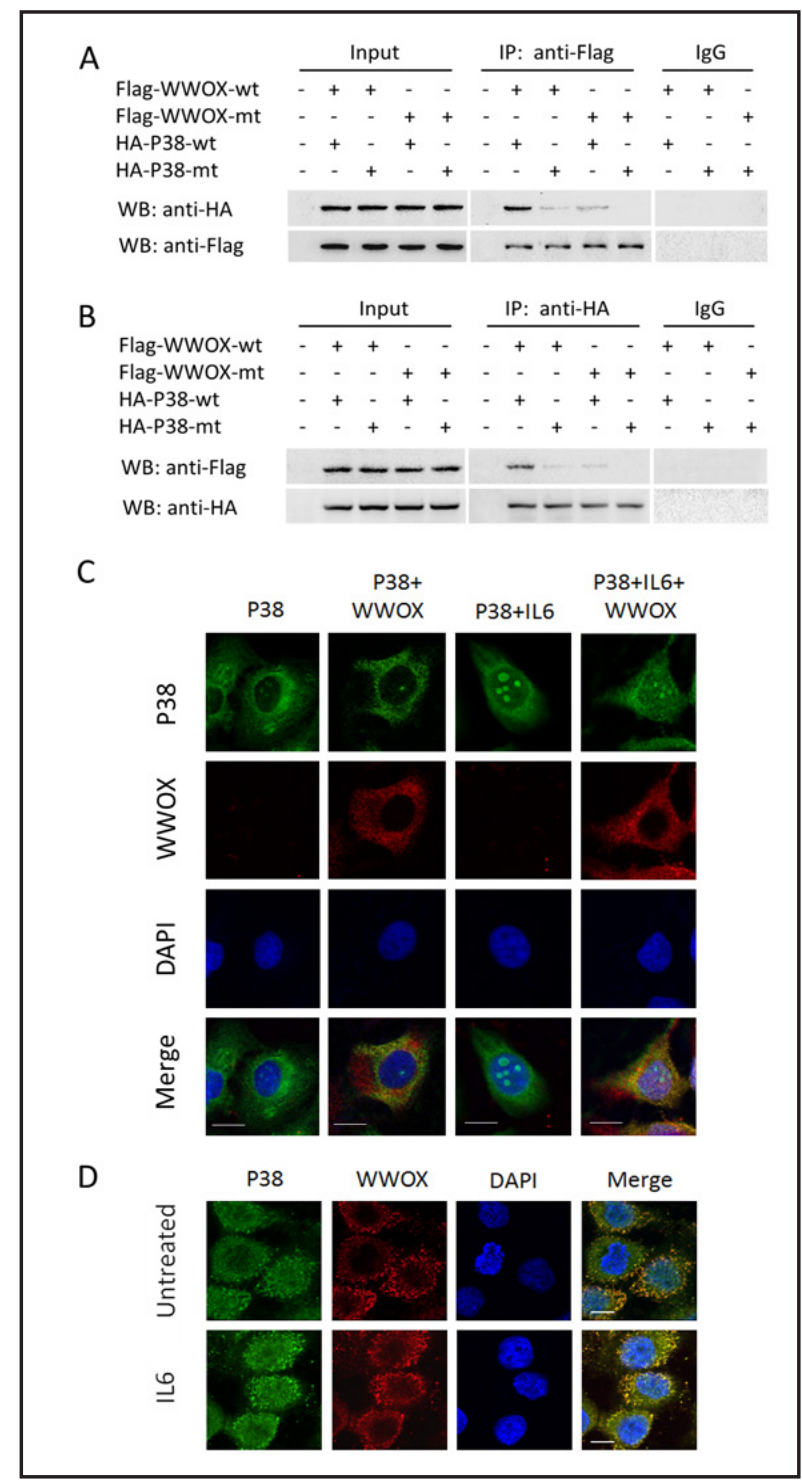

by sequestering in the cytoplasm through physical interactions. Therefore, we wanted to know whether WWOX modulated the sub-cellular localization of P38. As shown in Fig. 2E, endogenous P38 localized to the cytoplasm and nuclei of HuCCA-1 cells, suggesting an activated P38. When WWOX was over-expressed, however, the nuclear distribution of P38 decreased and P38 co-localized with WWOX in the cytoplasm, We found similar results in HEK293 cells (Fig. 1C). Additionally, IL-6-induced nuclear accumulation of P38 decreased when WWOX was introduced into HEK293 cells. To further confirm the effect of WWOX on p38 subcellular localization, we extracted the subcellular fractionation of HuCCA-1 cells and performed Western blotting. As shown in Fig. 2F and G.WWOX introduction caused dramatic decrease in nuclear distribution of P38. These data indicated that WWOX did not affect P38 expression and phosphorylation, but rather specifically inhibited the nuclear accumulation of P38.

WWOX inhibited the phosphorylation of ATF-2 and eIF-4E in a P38 MAPK-dependent manner

Activated P38 translocates to the nucleus, where it phosphorylates downstream transcriptional factors. ATF2 and eIF-4E are known P38 substrates that play important roles in tumorigenesis. Therefore, we tested the effect of WWOX on the phosphorylation 
Fig. 2. WWOX did not change the expression or phosphorylation state of P38, but it did alter its subcellular localization. [A] QBC939 cells were mocktreated or transfected with empty, wild type or mutant WWOX vector. Phosphorylated and total P38 was then detected after $24 \mathrm{~h}$. [B] Quantification of phosphorylated and total P38 in [A]. [C] RBE cells were mocktreated or transfected with empty, wild type or mutant WWOX vector, then treated $24 \mathrm{~h}$ later cells with IL-6 for an additional $6 \mathrm{~h}$, followed by detection of phosphorylated and total P38. [D] Quantification of phosphorylated or total P38 in [C]. [E] HuCCA-1 cells were mock-treated or transfected with wild or mutant type of WWOX plasmid, followed by immunofluorescence detection $24 \mathrm{~h}$ later. $\mathrm{Bar}=10$ $\mu \mathrm{m}$. [F] HuCCA-1 cells were treated as described in [E], and subcellular fractionation was extracted for Western blotting analysis. [G] Quantification of subcellular localization of P38 in [F]. N, nucleus. C, cytoplasm.

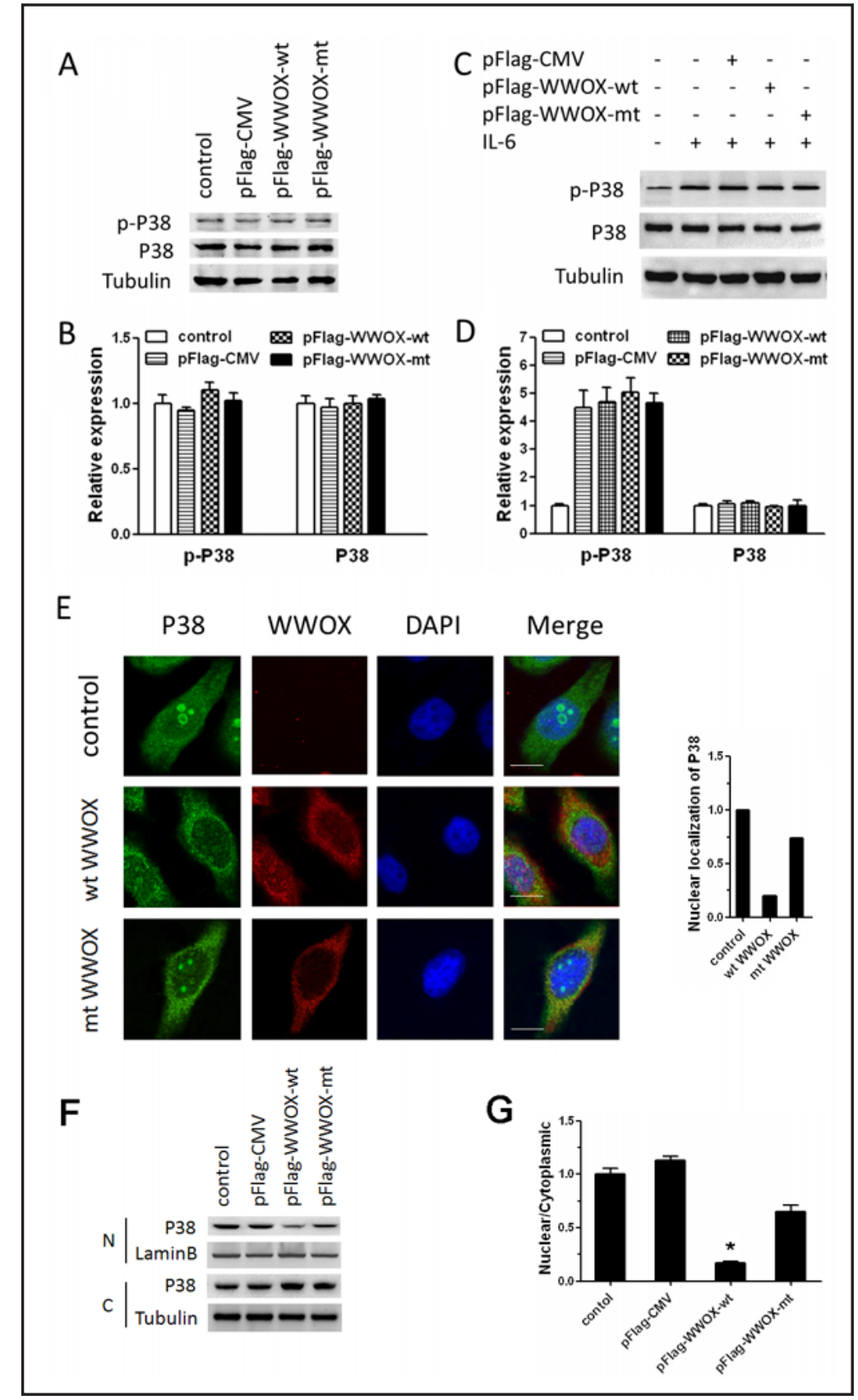

of these two molecules. As shown in Fig. 3A-C, over-expression of WWOX led to decreased levels of phosphorylated ATF2 and eIF-4E, an effect that was lost in the presence of mutant WWOX. Because mutated WWOX displayed impaired binding to P38, we performed rescue experiments to test the involvement of P38 in WWOX-induced down-regulation of ATF2 and eIF-4E phosphorylation. As shown in Fig. 3D and E, after introduction of P38, the reduction in ATF2 phosphorylation was restored to the level of WWOX-deficient cells. Phosphorylation of eIF-4E showed a similar trend after P38 was introduced (Fig. 3D and F). These results demonstrated that WWOX inhibited the phosphoylation of ATF2 and eIF-4E through P38 binding.

WWOX suppressed cell proliferation while inducing cell apoptosis via P38 MAPK

WWOX functions as a tumor suppressor, but there are no reports regarding its role in cholangiocarcinomas, particularly extrahepatic cholangiocarcinoma. In a previous study, we 
Fig. 3. WWOX inhibited the phosphorylation of ATF-2 and eIF-4E in a P38-dependent manner. [A] QBC939 cells were mock-treated or transfected with an empty, wild type or mutant WWOX vector, followed by detection of phosphorylated and total ATF-2 and eIF-4E 48 h later. [B] Quantification of phosphorylated/total ATF-2 in [A]. [C] Quantification of phosphorylated/total eIF-4E in [A]. [D] RBE cells were mock-treated or transfected with empty or WWOX vector, or co-transfected with WWOX plasmid together with empty or P38 vector. Phosphorylated and total ATF-2 and eIF-4E were detected after $48 \mathrm{~h}$. Quantification of phosphorylated/total protein is shown in [E] and [F]. *, p value is less than 0.05 vs. control.

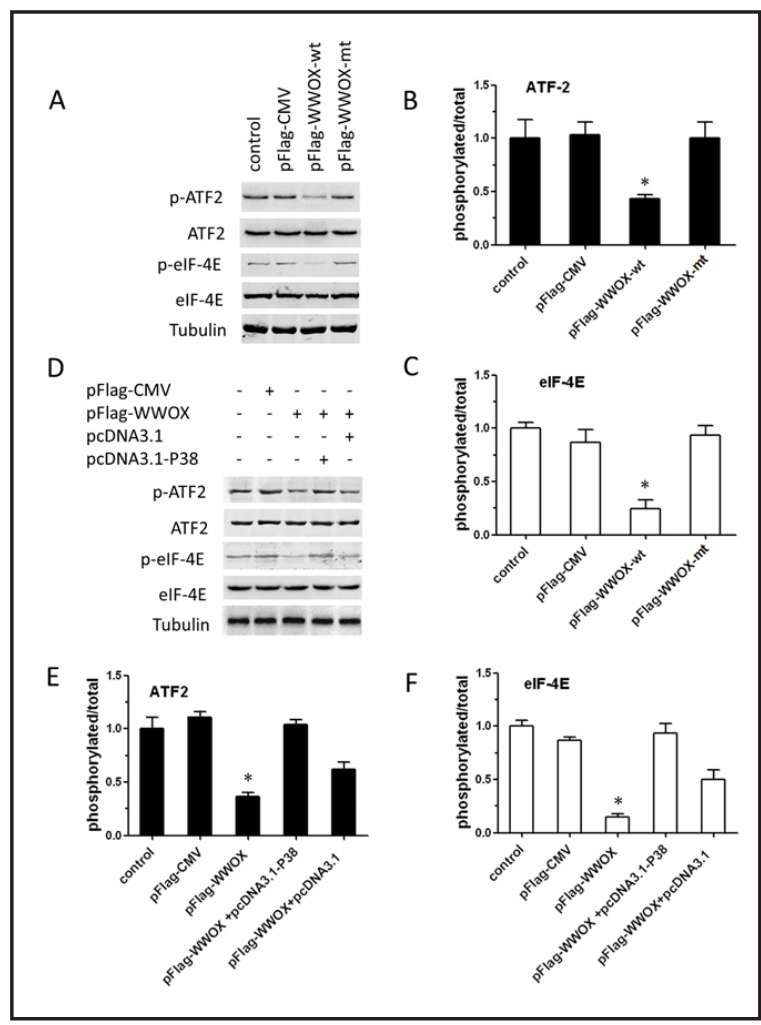

have demonstrated the decreased expression of WWOX in EHCC, but its specific role in EHCC was not defined. Therefore, here we examined the effect of WWOX on cell proliferation and apoptosis in EHCC. As shown in Fig. 4A, the cellular capacity for division was dramatically impaired after infected by WWOX adenovirus. As expected, this phenotype was partially rescued when both WWOX and P38 were over-expressed. A colony formation assay further confirmed the results obtained from the MTT assay (Fig. 4B). Based on our annexin V-FITC apoptosis assaym, over-expression of WWOX led to increased cell apoptosis, while P38 overexpression significantly decreased the apoptotic ratio (Fig. 4C). Additionally, the expression levels of the pro-apoptotic proteins Bax and Caspase- 3 were greatly increased after overexpression of WWOX. When P38 was introduced simultaneously with WWOX, the expression levels of Bax and Caspase-3 were elevated to a lesser extent than when the cells were in the presence of WWOX alone (Fig. 4D and E). Bcl-2, an anti-apoptotic protein, displayed the opposite expression pattern when the WWOX or P38 plasmids were introduced into QBC939 cells (Fig. 4D and E). These data demonstrated that WWOX suppressed cell proliferation and induced apoptosis in a P38 MAPK-dependent manner.

WWOX expression was negatively associated with phosphorylated ATF2 in EHCC tissues

Given that the phosphorylation of ATF2 and eIF-4E was down-regulated by WWOX, we next sought to detect phosphorylated ATF2 and eIF-4E in EHCC samples, and measured whether there was a clinical correlation between WWOX and ATF2 or eIF-4E in human tissue samples. We performed immunohistochemical analysis to assess the expression levels of WWOX, P38, and p-ATF2 or p-eIF-4E in 5 human normal bile duct samples and 30 EHCC samples. As shown in Fig. 5A, normal biliary duct tissue exhibited no positive staining for p-ATF2, but it did showe positive staining for WWOX. In contrast, the expression levels of p-ATF2 in EHCC samples were up-regulated compared to normal controls, whereas WWOX expression was lost in EHCC. P38 staining was positive in both normal and EHCC tissues. But more P38 localized in nucleus in EHCC compared with normal controls. The average foldchange of WWOX expression in EHCC was significantly lower than in normal bile duct samples (Fig. 5B), while that of nuclear P38 level and p-ATF2 expression in EHCC was significantly 
Fig. 4. WWOX suppressed cell proliferation while inducing cell apoptosis in a P38dependent manner. [A] RBE cells were mocktreated or infected with adenovirus containing EGFP or WWOX, and MTT assays were performed. [B] After infection with the indicated adenovirus, QBC939 cells were seeded in a 6-well plate for quantification of the colonies formed after $14 \mathrm{~d}$. [C] RBE cells were mocktreated or transfected with WWOX plasmid or WWOX plasmid plus P38 plasmid, and 48 h later, the Annexin V-FITC apoptosis assay was performed. [D] QBC939 cells were mock-treated or transfected with WWOX plasmid or WWOX plasmid plus P38 plasmid, followed by detection of apoptosis-associated protein $24 \mathrm{~h}$ later. [E] Quantification of protein expression. *, p value is less than 0.05 vs. control.

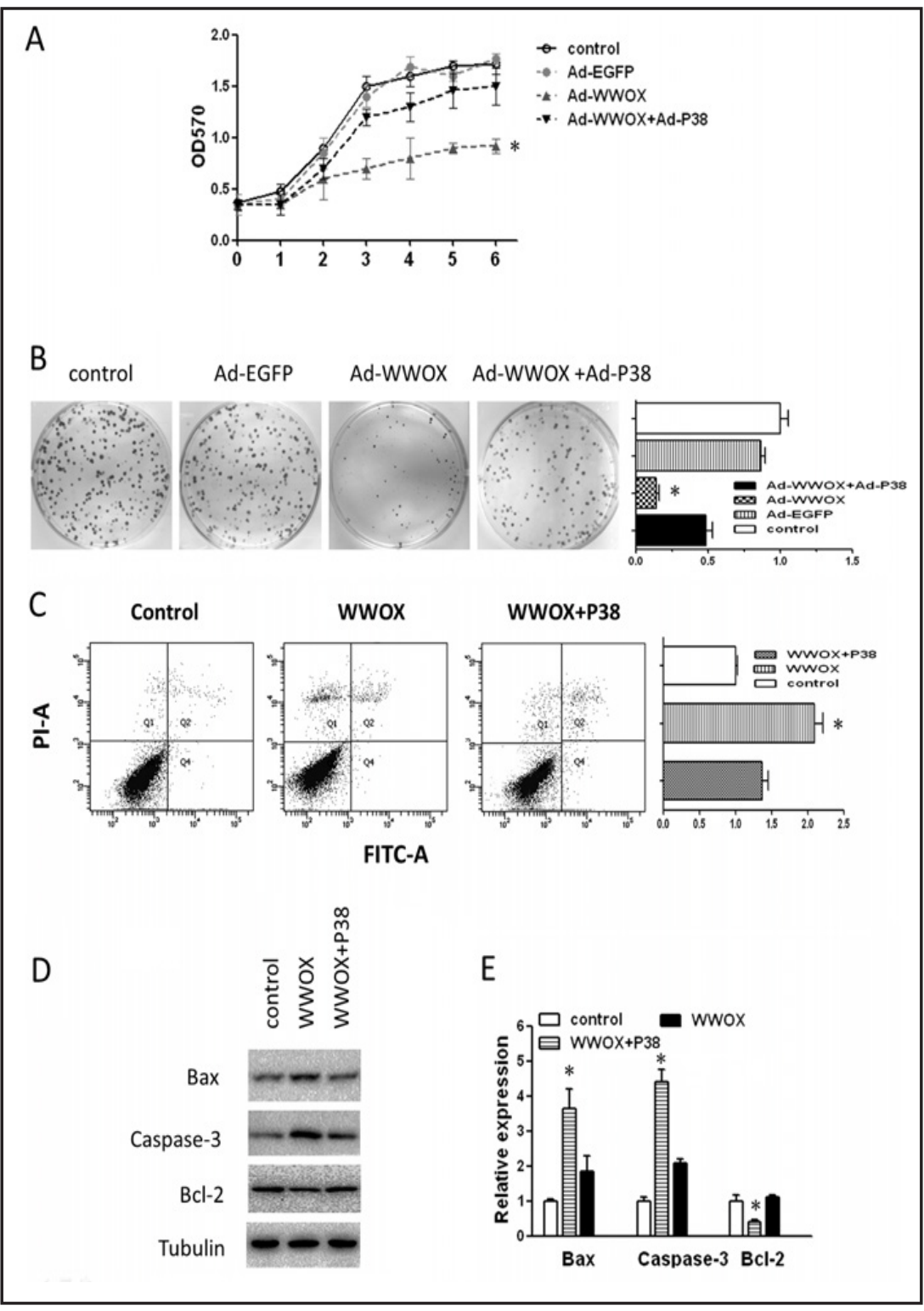

higher (Fig. 5C and D). Furthermore, an association study showed that nuclear P38 level and p-ATF2 expression negatively correlated with WWOX expression in these tissue samples (Fig. $5 \mathrm{E}$ and $\mathrm{F}$ ). These in vivo findings are consistent with previous in vitro observations that WWOX inhibits nuclear-localization of P38 as well as the phosphorylation of ATF2.

\section{Discussion}

EHCC is the second most common primary hepatic malignancy worldwide. Due to the poor prognosis, the majority of EHCC patients die within one year. Therefore, exploring the pathogenesis of EHCC, and finding effective markers for diagnosis and prognosis, as well as novel therapeutic target, is of great value for the treatment of EHCC.

Here, we demonstrated that WWOX interacted with P38 MAPK and prevented it from translocating to the nucleus and activating the transcriptional factors ATF2 and eIF-4E. WWOX suppressed EHCC cell proliferation and inducing apoptosis in a P38 MAPK-dependent manner. In EHCC samples, we found that the expression of WWOX was significantly lower relative to 
Fig. 5. WWOX expression was negatively associated with phosphorylated ATF2 in normal and EHCC tissues. [A] Immunohistochemical staining of WWOX, P38, and p-ATF2 in human normal biliary duct and EHCC samples. [B] - [D] Relative expression levels of WWOX, nulear P38, and p-ATF2 and in human normal and EHCC samples. *, p value is less than 0.05 vs. control. [E] and [F] Correlation between WWOX and nuclear P38/pATF2 expression with linear regression lines and Pearson's correlation significance $[\mathrm{P}$ value is less than 0.001 , analysis of variance test].

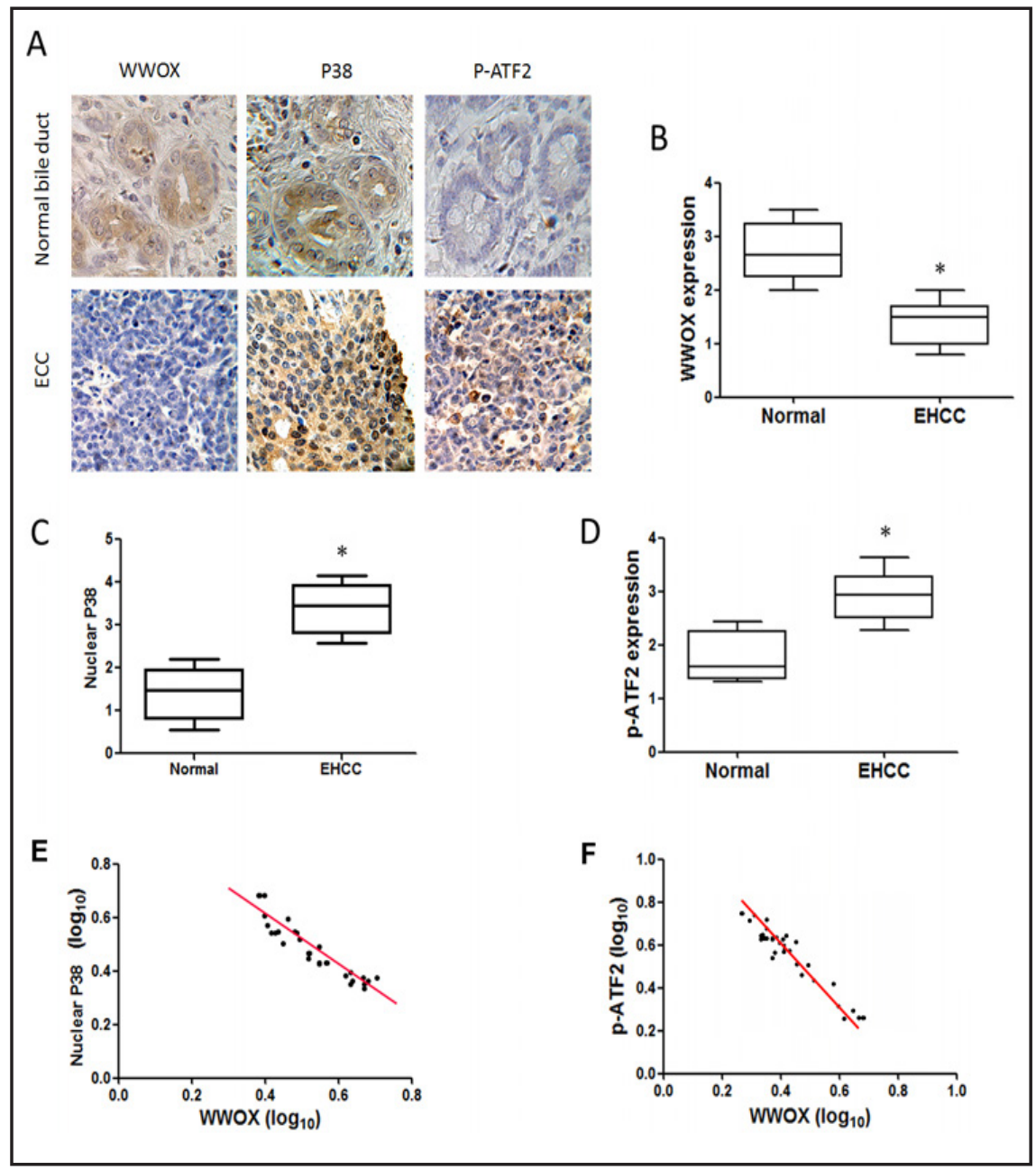

normal control tissues, and it was negatively correlated with the level of phosphorylated ATF2. These results suggested that the WWOX-pATF2 correlation may have prognostic value. The WWOX-p38-ATF2 pathway elucidated in this study is likely an important component of the underlying mechanisms of EHCC tumorigenesis and progression. To our knowledge, this is the first study reporting the role of WWOX in EHCC. Targeting strategies based on the newly discovered WWOX-p38-ATF2 pathway may be exploited in the near future.

WWOX contains two WW domains in its N-terminal region. The first associates with the P-P-X-Y motif of a number of transcription factors, leading to the suppression of transcriptional activities by sequestering these proteins in the cytoplasm. Therefore, the role of WWOX in various signal transduction pathways is indirect. Until now, there have been no reports that defined P38 MAPK as a WWOX interacting partner. Our results here revealed that P38 is a WWOX-associated protein, expanding the current understanding of WWOXmediated signaling. In addition to the N-terminal WW domains, the C-terminus of WWOX contains a short-chain alcohol dehydrogenase reductase SDR] domain, which is involved in regulation of metabolic steroids activities. We did not involve the SDR domain in our study because EHCC is not hormone-dependent.

The stability and sub-cellular localization of WWOX are regulated by its phosphorylation. Tyr33 and Tyr287 are the known phosphorylation sites of WWOX. Tyr33 phosphorylation can induce the nuclear translocation of WWOX and inhibit its ability to bind to interacting partners. In contrast, Tyr287 phosphorylation is associated with poly-ubiquitination and degradation. However, the interaction between WWOX and P38 did not affect the 
phosphorylation state of WWOX, including at Tyr33 and Tyr287, leading to no change in either the cytoplasmic localization or abundance of WWOX [data not shown].

P38 MAPK has been implicated in the tumorigenesis of EHCC. The P38 signaling pathway is a notable molecular feature of this cancer, and utilizing therapeutic targets and inhibitors of P38 MAPK, such as SB203580, will undoubtedly be key strategies for EHCC treatment [31]. Importantly, our study elucidated a novel and effective mechanism to modulate the activity of P38. That is, altering the sub-cellular localization of P38 by introducing WWOX, with the goal of attenuating its phosphorylation of transcriptional factors in the nucleus. In addition, although the phosphorylation and activation of P38 are both necessary for its nuclear translocation, WWOX did not regulate the phosphorylation of P38, but rather only sequestered it in the cytoplasm. It has been reported that P38 has at least 4 isoforms. In this study, all of the analyses were performed with P38 alpha. In addition to the alpha isoform, beta, gamma, and delta isoforms of P38 also exist. We analyzed the sequence of other isoforms of P38, and did not find PPXY motif in them. Theoretically, WWOX specifically binds alpha P38.

ATF2 is expressed ubiquitously and can form either homodimers or heterodimers with c-Fos, Fra2 and c-Jun to transcriptionally activate target gene expression by binding to CRE [cAMP response element] consensus sequences or to activator protein 1 [AP-1] consensus sequences. The tumor-promoting role of ATF2 has been implicated in several types of cancer, such as melanoma and skin carcinoma [32]. Here, our data suggested for the first time the involvement of ATF2 in EHCC, as phosphorylation of ATF2 was increased in EHCC samples. This result provides the possibility of exploiting p-ATF2 as a useful prognostic marker. The high level of phosphorylated ATF2 in EHCC was most likely due to the deficiency of WWOX. In addition to regulation by phosphorylation, ATF2 activity can also be regulated by mechanisms such as its sub-cellular localization and the maintenance of stability. Our study did not investigate the effect of WWOX and P38 on sub-cellular localization or stability of ATF2, therefore, further experiments will be needed in the future. ATF7, another member of the activating transcription factor [ATF] family, is structurally similar to ATF2, particularly within the basic region/leucine zipper [bZIP] DNA-binding and dimerization domains. However, we did not find the same results with of ATF7 as with ATF2 in our system, suggesting distinct roles for these proteins EHCC [data not shown]. Further investigation is needed to better understand the differences between these two family members.

Taken together, our data in this study revealed that WWOX interacts with P38 MAPK, leading to the cytoplasmic retention of P38 and inhibition of ATF 2 and eIF-4E phosphorylation. Ectopic expression of WWOX reduced proliferation and induced apoptosis in EHCC cells. Additionally, WWOX expression was significantly lower and negatively correlated with phosphorylated ATF2 levels in EHCC tissues, compared to normal samples. To our knowledge, this is the first study reporting the role and underlying mechanism of WWOX in EHCC. Our data not only expand the current understanding of WWOX-mediated signaling, but they also provid the possibility of exploiting WWOX/p-ATF2 as a useful diagnostic and prognostic marker, as well as a novel therapeutic target for the treatment of EHCC.

\section{Disclosure Statement}

None.

\section{Acknowledgments}

We thank Lizhi Wang of The Third Military Medical University for providing QBC939, RBE and HuCCA-1 cholangiocarcinoma cells 
Wang et al.: WWOX Inhibits P38 to Suppress Cholangiocarcinoma Cell Growth

\section{References}

1 Razumilava N, Gores GJ: Cholangiocarcinoma. Lancet 2014;383:2168-2179.

2 Rizvi S, Gores GJ: Pathogenesis, diagnosis, and management of cholangiocarcinoma. Gastroenterology 2013;145:1215-1229.

3 Al-Bahrani R, Abuetabh Y, Zeitouni N, Sergi C: Cholangiocarcinoma: risk factors, environmental influences and oncogenesis. Ann Clin Lab Sci 2013;43:195-210.

4 Friman S: Cholangiocarcinoma--current treatment options. Scand J Surg 2011;100:30-34.

5 Sawanyawisuth K: Genes and cholangiocarcinoma. Southeast Asian J Trop Med Public Health 2009;40:701712.

6 Wong TC, Poon RT: Hepatobiliary malignancies: lessons from Asia. Dig Dis 2013;31:130-137.

7 Salah Z, Aqeilan R, Huebner K: WWOX gene and gene product: tumor suppression through specific protein interactions. Future Oncol 2010;6:249-259.

8 Yang J, Zhang W: WWOX tumor suppressor gene. Histol Histopathol 2008;23:877-882.

99 Aqeilan RI, Croce CM: WWOX in biological control and tumorigenesis. J Cell Physiol 2007;212:307-310.

10 Hezova R, Ehrmann J, Kolar Z: WWOX, a new potential tumor suppressor gene. Biomed Pap Med Fac Univ Palacky Olomouc Czech Repub 2007;151:11-15.

-11 Xiong A, Wei L, Ying M, Wu H, Hua J, Wang Y: Wwox suppresses breast cancer cell growth through modulation of the hedgehog-GLI1 signaling pathway. Biochem Biophys Res Commun 2014;443:1200-1205.

12 Hong Q Sze CI, Lin SR, Lee MH, He RY, Schultz L, Chang JY, Chen SJ, Boackle RJ, Hsu LJ, Chang NS: Complement C1q activates tumor suppressor WWOX to induce apoptosis in prostate cancer cells. PLoS One 2009;4:e5755.

13 Becker S, Markova B, Wiewrodt R, Hoffarth S, Hähnel PS, Pleiner S, Schmidt LH, Breitenbuecher F, Schuler M: Functional and clinical characterization of the putative tumor suppressor WWOX in non-small cell lung cancer. J Thorac Oncol 2011;6:1976-1983.

14 Lin JT, Tzai TS, Liao CY, Wang JS, Wu TT, Wang HY, Wu CH, Yu CC, Lu PJ: WWOX protein expression varies among RCC histotypes and downregulation of WWOX protein correlates with less-favorable prognosis in clear RCC. Ann Surg Oncol 2013;20:193-199.

15 Nakayama S, Semba S, Maeda N, Matsushita M, Kuroda Y, Yokozaki H: Hypermethylation-mediated reduction of WWOX expression in intraductal papillary mucinous neoplasms of the pancreas. Br J Cancer 2009;100:1438-1443.

16 Aqeilan RI, Palamarchuk A, Weigel RJ, Herrero JJ, Pekarsky Y, Croce CM: Physical and functional interactions between the Wwox tumor suppressor protein and the AP-2gamma transcription factor. Cancer Res 2004;64:8256-8261.

17 Lin D, Cui Z, Kong L, Cheng F, Xu J, Lan F: p73 participates in WWOX-mediated apoptosis in leukemia cells. Int J Mol Med 2013;31:849-854.

18 Gomes CC, Diniz MG, Oliveira CS, Tavassoli M, Odell EW, Gomez RS, De Marco L: Impact of WWOX alterations on $\mathrm{p} 73, \Delta \mathrm{Np} 73, \mathrm{p} 53$, cell proliferation and DNA ploidy in salivary gland neoplasms. Oral Dis 2011;17:564-571.

19 Aqeilan RI, Donati V, Palamarchuk A, Trapasso F, Kaou M, Pekarsky Y, Sudol M, Croce CM: WW domaincontaining proteins, WWOX and YAP, compete for interaction with ErbB-4 and modulate its transcriptional function. Cancer Res 2005;65:6764-6772.

20 Gaudio E, Palamarchuk A, Palumbo T, Trapasso F, Pekarsky Y, Croce CM, Aqeilan RI: Physical association with WWOX suppresses c-Jun transcriptional activity. Cancer Res 2006;66:11585-44589.

-21 Yang J, Zhao L, Tian W, Liao Z, Zheng H, Wang G, Chen K: Correlation of WWOX, RUNX2 and VEGFA protein expression in human osteosarcoma. BMC Med Genomics 2013;6:56.

22 Del Mare S, Kurek KC, Stein GS, Lian JB, Aqeilan RI: Role of the WWOX tumor suppressor gene in bone homeostasis and the pathogenesis of osteosarcoma. Am J Cancer Res 2011;1:585-594.

23 Kurek KC, Del Mare S, Salah Z, Abdeen S, Sadiq H, Lee SH, Gaudio E, Zanesi N, Jones KB, DeYoung B, Amir G, Gebhardt M, Warman M, Stein GS, Stein JL, Lian JB, Aqeilan RI: Frequent attenuation of the WWOX tumor suppressor in osteosarcoma is associated with increased tumorigenicity and aberrant RUNX2 expression. Cancer Res 2010;70:5577-5586. 
24 Aqeilan RI, Hassan MQ, de Bruin A, Hagan JP, Volinia S, Palumbo T, Hussain S, Lee SH, Gaur T, Stein GS, Lian JB, Croce CM: The WWOX tumor suppressor is essential for postnatal survival and normal bone metabolism. J Biol Chem 2008;283:21629-21639.

25 Cargnello M, Roux PP: Activation and function of the MAPKs and their substrates, the MAPK-activated protein kinases. Microbiol Mol Biol Rev 2011;75:50-83.

-26 Arthur JS, Ley SC: Mitogen-activated protein kinases in innate immunity. Nat Rev Immunol 2013;13:679692.

27 Munshi A, Ramesh R: Mitogen-Activated Protein Kinases and Their Role in Radiation Response. Genes Cancer 2013;4:401-408.

-28 Koul HK, Pal M, Koul S: Role of p38 MAP Kinase Signal Transduction in Solid Tumors. Genes Cancer 2013;4:342-359.

29 del Barco Barrantes I, Nebreda AR: Roles of p38 MAPKs in invasion and metastasis. Biochem Soc Trans 2012;40:79-84.

30 Wang M1, Gu J, Wang Y, Gong B: Loss of WWOX expression in human extrahepatic cholangiocarcinoma. J Cancer Res Clin Oncol 2009;135:39-44.

-31 Wong P, Hsieh F, Pham R, James CA: Critical review of p38 MAP kinase inhibitors: a bioanalytical perspective. Bioanalysis 2012;4:89-93.

-32 Gozdecka M, Breitwieser W: The roles of ATF2 [activating transcription factor 2] in tumorigenesis. Biochem Soc Trans 2012;40:230-234. 